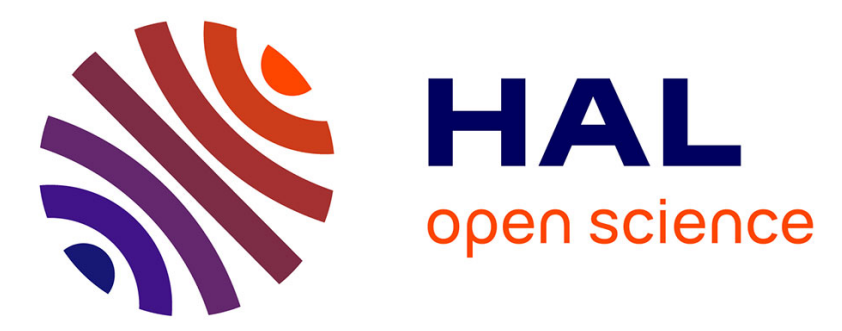

\title{
Reliability analysis of Markov history-dependent repairable systems with neglected failures
}

\author{
Shijia Du, Zhiguo Zeng, Lirong Cui, Rui Kang
}

\section{To cite this version:}

Shijia Du, Zhiguo Zeng, Lirong Cui, Rui Kang. Reliability analysis of Markov history-dependent repairable systems with neglected failures. Reliability Engineering and System Safety, 2017, 159, pp.134-142. 10.1016/j.ress.2016.10.030 . hal-02447684

\section{HAL Id: hal-02447684 \\ https://hal.science/hal-02447684}

Submitted on 24 Feb 2020

HAL is a multi-disciplinary open access archive for the deposit and dissemination of scientific research documents, whether they are published or not. The documents may come from teaching and research institutions in France or abroad, or from public or private research centers.
L'archive ouverte pluridisciplinaire HAL, est destinée au dépôt et à la diffusion de documents scientifiques de niveau recherche, publiés ou non, émanant des établissements d'enseignement et de recherche français ou étrangers, des laboratoires publics ou privés. 


\title{
Reliability analysis of Markov history-dependent repairable systems
}

\author{
with neglected failures
}

\author{
Shijia Du ${ }^{\mathrm{a}}$, Zhiguo Zeng a ${ }^{{ }^{*}}$, Lirong Cui ${ }^{\mathrm{b}}$, Rui Kang ${ }^{\mathrm{a}}$ \\ ${ }^{\text {a }}$ School of Reliability and Systems Engineering, Beihang University, Beijing 100191, China \\ ${ }^{\mathrm{b}}$ School of Management and Economics, Beijing Institute of Technology, Beijing, 100081, China
}

\begin{abstract}
:
Markov history-dependent repairable systems refer to the Markov repairable systems in which some states are changeable and dependent on recent evolutional history of the system. In practice, many Markov history-dependent repairable systems are subjected to neglected failures, i.e., some failures do not affect system performances if they can be repaired promptly. In this paper, we develop a model based on the theory of aggregated stochastic processes to describe the history-dependent behavior and the effect of neglected failures on the Markov history-dependent repairable systems. Based on the developed model, instantaneous and steady-state availabilities are derived to characterize the reliability of the system. Four reliability-related time distributions, i.e., distribution for the $k$ th working period, distribution for the $k$ th failure period, distribution for the real working time in an effective working period, distribution for the neglected failure time in an effective working period, are also derived to provide a more comprehensive description of the system's reliability. Thanks to the power of the theory of aggregated stochastic processes, closed-form expressions are obtained for all the reliability indexes and time distributions. Finally, the developed indexes and analysis methods are demonstrated by a numerical example.
\end{abstract}

Keywords: Markov repairable system; Markov history-dependent repairable systems; neglected failure; availability; aggregated stochastic process

\section{Introduction}

Reliability is an important feature of repairable systems [1]-[4]. In most existing literatures, reliability analysis is studied by modeling the repairable system as a Markov process (e.g., see [5]-[7]). Most of these Markov-process-based models assume that there are clear definitions of normal state and failure state and the definitions are independent of the evolutional history of the system. In practice, however, some states in a repairable system might be changeable and depend on the recent evolutional history of the system (for example, see [8] [9]). This kind of special system was named by Cui et al. [8] as Markov Repairable System with History-Dependent up and down States (MRS-HDS) and reliability analysis methods were developed by them to calculate its availability. Based on the work of [8], Wang et al. [10] derived multi-point and multi-interval availabilities for the MRS-HDS. Zheng et al. [11] generalized Cui's work in [8] to semi-Markov repairable systems with history-dependent up and down states.

In many practical systems, if the repair time is shorter than a predefined threshold, the failures will have little influence on systems' performances, so that the failures can be neglected

${ }^{*}$ Corresponding author, Email: zengzhiguo@ buaa.edu.cn 
when evaluating the system's availability. For example, consider a failure in a water supply system that cut off the water supply to the end-users. If the failure is recovered in a relatively short period, e.g., several minutes, the end-users are possible to tolerate or even unaware of it. This kind of failure, which can be promptly repaired within the tolerance of the end-users, can therefore, be neglected when calculating the availability of the water supply system. Such failures are regarded as neglected failures and are commonly encountered in repairable systems. For instance, Zheng et al. [12] studied a single-unit Markov repairable system with repair time omission. Bao and Cui [13] considered series Markov repairable systems with neglected or delayed failures.

Neglected failures also exist in MRS-HDS, e.g. the water distributed system, the power distributed networks, computer networks, etc. The introduction of changeable states, as a result of the neglected failures, makes standard Markov-process-based models incapable to capture the system's failure and repair behaviors. However, to the best of our knowledge, the effect of neglected failures on the reliability of MRS-HDS has not been considered before. To fill this gap, we develop a new model and associated reliability analysis method based on the theory of aggregated stochastic process and then derive some important reliability indexes for the system. Although a large number of literatures focused on obtaining reliability indexes for repairable systems (e.g. [14]-[16]), it is generally difficult to obtain close-formed expressions for these indexes, especially when the system's behavior is complex, as the case of MRS-HDS with neglected failures. In this paper, however, with the help of the aggregated stochastic processes theory, close-formed expressions can be obtained for all the derived indexes.

Aggregated stochastic processes were invented by Burke and Rosenblatt [17] in 1957. One of the milestones in the history of the aggregated stochastic process is Colquhoun and Hawkes's successful work in developing probabilistic models to describe the behavior of ion channels (e.g., see [18] [19]). Since then, aggregated stochastic process has received more and more attention from various areas. Among them, Rubino and Sericola [20] first applied the theory to model a repairable system. Various models were, then, developed based on the aggregated stochastic processes to describe the behavior of different types of repairable systems, e.g., Hawkes et al. (e.g., [21]), Cui et al. (e.g. [22] and [23]), Wang et al. ([24] and [25]), Liu et al. (e.g. [26]), etc. By employing aggregated stochastic processes, we focus on aggregating and decomposing the states of the new model. To be specific, because changeable and down states can present both failure and operational states, these two states are duplicated and redefined, in order to describe the state-dependent and time-dependent behavior of the system. A new stochastic process, which is an aggregated stochastic process, can be constructed and the theory of aggregated processes can be used to achieve analytical expressions of some important reliability indexes and reliability-related time distributions.

The rest of the paper is organized as follows. In Section 2, the motivation of this paper is fully explained with an industrial example and assumptions of the developed model are formally presented. In Section 3, we use aggregated stochastic processes to model the behavior of MRS-HDS with neglected failures. Some reliability indexes for MRS-HDS with neglected failures, i.e., the instantaneous availability, steady-state availability and some sojourn time distributions are derived in Section 4. In Section 5, the developed indexes and analysis methods are demonstrated by a numerical example. Finally, conclusions are given in Section 6. 


\section{Motivation and assumptions}

\subsection{Motivation example}

In this section, we present an engineering example of MRS-HDS with neglected failures which motivates the research in this paper. Take an electrical power supply system as an example, which is designed to provide electricity to a factory. The electricity comes from three substations, each of which can contribute a maximum amount of $1 \mathrm{MW}$ (Megawatt). To maintain its normal functions, the factory needs a total amount of $2.5 \mathrm{MW}$ of electricity. Due to the advance technology of distributed power generations [27] [28], the factory itself can produce $0.8 \mathrm{MW}$ of electricity since it has installed a photovoltaic generator. However, the photovoltaic generator only works when the factory is in normal state, since it requires power supplies and control commands from a control center, which is located in the factory. If the factory does not receive enough power from the electricity-distributed system, the control center cannot work, which in turn, ceases the normal functioning of the photovoltaic generator.

The state space of the system can be defined by the numbers of the failed substations: $S=\{0,1,2,3\}$. It is easy to verify that when the system is in state 0 , the factory is in working state; when the system is in state 2 or state 3 , the factory is in failure state, regardless of the state of the photovoltaic generator. However, state 1, which represents that one of the three substations fails and the factory receives $2 \mathrm{MW}$ of electricity from the electricity supply system, is a changeable state affected by the system's historical dynamics:

(1) Suppose the system transfers to state 1 from a working state, which means that before entering state 1 , the factory was functioning normally. Hence, the photovoltaic generator is working and generates additional $0.8 \mathrm{MW}$ of electricity. The total amount of electricity the factory could receive is, then, calculated as $2+0.8=2.8(\mathrm{MW}) \geq 2.5(\mathrm{MW})$. Therefore, the factory can perform its function normally. It means state 1 of the system is a working state.

(2) If, on the other hand, the system transfers to state 1 from a failure state (state 2 or state 3 ), which means that before entering state 1 , the factory was already in failure state, and therefore, the photovoltaic generator cannot work. The factory can, then, only receive total amount of 2 MW electricity, which makes it in a failure state.

Consequently, state 1 in the above electrical power supply system is a history-dependent state. Besides, due to the widely application of Uninterrupted Power Supplies (UPSs) in the factory, the factory can withstand a temporary loss-of-power for no more than 5 minutes. Thus, failures that can be recovered within 5 minutes are treated as neglected failures. In this paper, we develop methods to analyze the reliability and availability of such MRS-HDS with neglected failures.

\subsection{System descriptions and assumptions}

In this section, we present a formal description of MRS-HDS with neglected failures. The system is based on the following assumptions:

(1) The repairable system is described by a continuous-time Markov process $\{X(t), t \geq 0\}$ with finite state space $S$.

(2) The observation for the system has two statuses: Up and Down periods. Essentially, the state space $S$ can be divided into three sets, $U, C$, and $D$. The state in $U$ stands for 
functional or Up state, which represents that the system is working. When the system stays in $U$, we say that the system is in Up period. The state in $D$ stands for failure or Down state, which represents that the system fails. When the system stays in $D$, we say that the system is in Down period. The state in $C$ represents changeable state.

(3) The changeable states in $C$ could be observed to be either in Up or Down period, depending on the system's historical state-transition trajectory: If the last non-changeable state prior to the changeable state is in Up period, then the changeable state is classified as an Up state, referred to as changeable-up states, denoted as $C_{U}$. The system will be in Up period when it stays in $C_{U}$. If, on the other hand, the last non-changeable state prior to the changeable state is in Down period, then the changeable state is classified as a Down state, referred to as changeable-down states, denoted as $C_{D}$. The notations for changeable states are the same as that in [8], which is illustrated in Figure 1.

(4) If the repair time in $D$, denoted by $t_{R}$, is longer than a predefined threshold $\tau, t_{R}$ is still regarded as in Down period; if, on the other hand, $t_{R}$ is less than $\tau$, failures in $D$ is neglected: If the previous state belongs to Up period, the system is regarded as in Up period for the whole period of $t_{R}$; on the other hand, if the previous state belongs to Down period, then the system is regarded as in Down period for the whole period of $t_{R}$.

Therefore, the rule to judge which period the system stays in is to know what period the previous state belongs to. According to this rule, the illustration of MRS-HDS with neglected failures is shown in Figure 2.

Here, we want to mention that the definition of neglected failure is adopted from the definition in [12]. According to [12], when the system comes from "up" to "down" with a duration longer than $\tau$, the whole duration of repair (or failure) is regarded as in "down". Only when the duration of repair time for the system is no longer than $\tau$, the "down" duration will be considered to be "operating" (available). That is to say, when the system comes from "up state" to a long $D$ state, the first $\tau$ time units will be considered as part of downtime. The reason we made explanation is to distinguish two different understanding for the availability of the first $\tau$ time units in a long $D$ state. The definition used in our model is based on model II in [23], which is called the neglected failure model. However, if the first $\tau$ time units in a long $D$ state is regarded as available, the model will become another one which is same as model III in [23], called failure delayed failure model. 


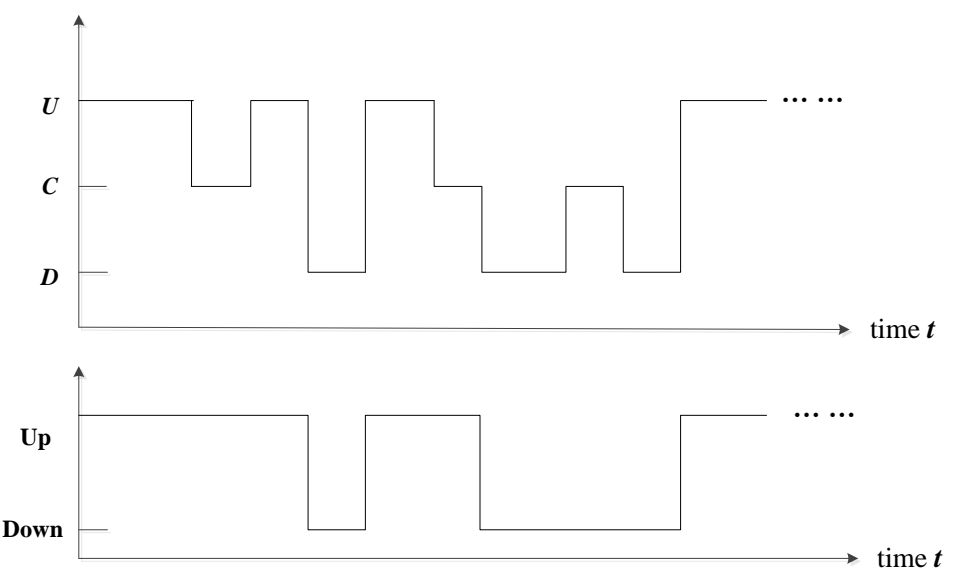

Figure 1. A illustration of changeable states

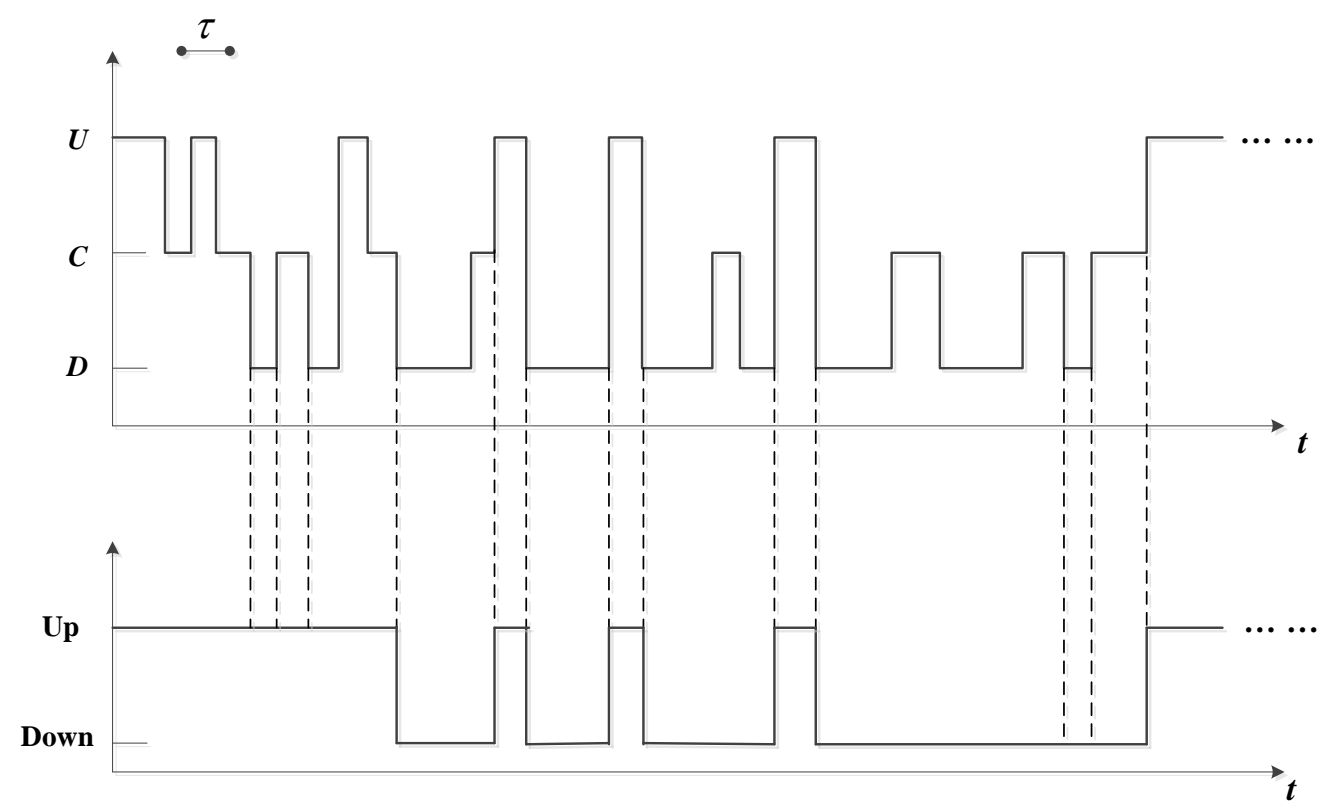

Figure 2. A illustration of MRS-HDS with neglected failures

\section{Modelling MRS-HDS with neglected failures using aggregated stochastic processes}

In this section, we use aggregated stochastic processes, a mathematical tool mainly developed in the theory of ion channel, to model the behaviour of MRS-HDS with neglected failures. First, the Markov process in which the finite number of states is partitioned into three sets, $U, C$, and $D$ has transition rate matrix

$$
\boldsymbol{Q}=\left(\begin{array}{lll}
\boldsymbol{Q}_{U U} & \boldsymbol{Q}_{U C} & \boldsymbol{Q}_{U D} \\
\boldsymbol{Q}_{C U} & \boldsymbol{Q}_{C C} & \boldsymbol{Q}_{C D} \\
\boldsymbol{Q}_{D U} & \boldsymbol{Q}_{D C} & \boldsymbol{Q}_{D D}
\end{array}\right) .
$$

Then, a semi-Markov process can be defined, which is imbedded at the instants when the system switches between the three groups. The stochastic properties of this semi-Markov process are governed by a transition matrix $\boldsymbol{G}(t)$, which can be partitioned in a similar manner to $\boldsymbol{Q}$ as 


$$
\boldsymbol{G}(t)=\left(\begin{array}{ccc}
\boldsymbol{0} & \boldsymbol{G}_{U C}(t) & \boldsymbol{G}_{U D}(t) \\
\boldsymbol{G}_{C U}(t) & \boldsymbol{0} & \boldsymbol{G}_{C D}(t) \\
\boldsymbol{G}_{D U}(t) & \boldsymbol{G}_{D C}(t) & \boldsymbol{0}
\end{array}\right),
$$

where $\boldsymbol{G}_{\alpha \beta}(t)=\exp \left(\boldsymbol{Q}_{\alpha \alpha} t\right) \boldsymbol{Q}_{\alpha \beta}$, provided $\alpha \neq \beta$, for $\alpha, \beta=U, C$, or $D$.

\subsection{Modelling the history-dependent behaviour}

Next, from another viewpoint, once the system enters one $U$ state, then the system is sojourn in Up period until one long $D$ duration (duration in $D$ longer than the given value) occurs. More specifically, no matter what transitions happening after that $U$ state, the system is always in Up period as long as no arriving in long $D$ duration.

Similarly, the system is sojourn in Down period once it comes to one $D$ duration longer than the given value. And the Down period will terminate when one $U$ state occurs. The following diagram, Figure 3 can illustrate this viewpoint.
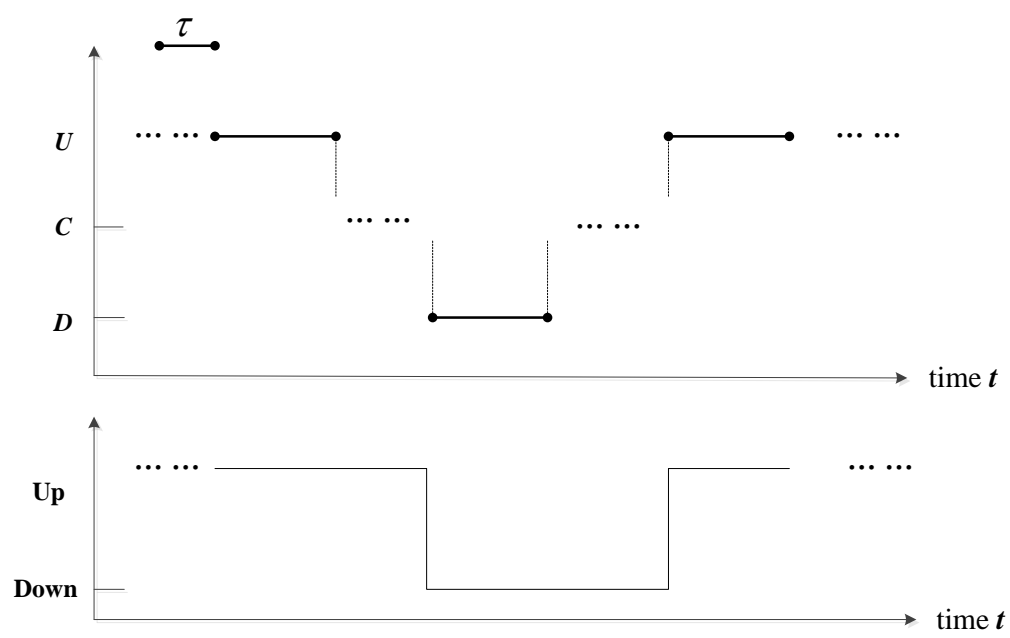

Figure 3. Alternative Up and Down periods path for the system

If we use stochastic process $\{X(t), t \geq 0\}$ to describe the running path for the system, then another stochastic process $\{Y(t), t \geq 0\}$, can be seen as the results of our observation. That is whether the system is in Up or Down period. The stochastic process $\{Y(t), t \geq 0\}$ is an aggregated stochastic process.

If we denote the Up period as $W$, Down period as $F$, then the properties of this system may be described by considering a semi-Markov process. That is to say, the aggregated stochastic process $\{Y(t), t \geq 0\}$ is a semi-Markov process.

The semi-Markov process has a density kernel, which will be denoted by 


$$
{ }^{e} \overline{\boldsymbol{G}}(t)=\left(\begin{array}{cc}
\boldsymbol{0} & { }^{e} \overline{\boldsymbol{G}}_{W F}(t) \\
e \overline{\boldsymbol{G}}_{F W}(t) & \boldsymbol{0}
\end{array}\right) .
$$

For some purposes, it is useful to have their Laplace transforms:

$$
{ }^{e} \overline{\boldsymbol{G}}^{*}(s)=\left(\begin{array}{cc}
\boldsymbol{0} & { }^{e} \overline{\boldsymbol{G}}_{W F}^{*}(s) \\
e \overline{\boldsymbol{G}}_{F W}^{*}(s) & \boldsymbol{0}
\end{array}\right) .
$$

If we ignore the durations of the sojourns and merely consider the sequence of states that are occupied at the start of each sojourn, we have an embedded Markov chain with transition probability matrix,

$$
{ }^{e} \overline{\boldsymbol{G}}=\left(\begin{array}{cc}
\boldsymbol{0} & { }^{e} \overline{\boldsymbol{G}}_{W F} \\
\overline{\boldsymbol{G}}_{F W} & \boldsymbol{0}
\end{array}\right)=\int_{0}^{\infty e} \overline{\boldsymbol{G}}(t) d t={ }^{e} \overline{\boldsymbol{G}}^{*}(0) .
$$

Here we use the convention of dropping the star and the argument to denote a Laplace transform evaluated at $s=0$, which is the integral of the original function over the interval $(0, \infty)$.

\subsection{Modelling the neglected failures}

Taking neglected failure into consideration, we need to distinguish short (less than $\tau$ ) and long (greater than $\tau$ ) sojourns in $D$. So in the manner of Hawkes et al. [29], we break up the Laplace transform of $\boldsymbol{G}_{D C}^{*}(s)$ into two parts as follows.

$$
\begin{gathered}
\int_{0}^{\tau} e^{-s t} \exp \left(\boldsymbol{Q}_{D D} t\right) \boldsymbol{Q}_{D C} d t=\left(\boldsymbol{I}-\exp \left(-\left(s \boldsymbol{I}-\boldsymbol{Q}_{D D}\right) \tau\right)\right)\left(s \boldsymbol{I}-\boldsymbol{Q}_{D D}\right)^{-1} \boldsymbol{Q}_{D C}=\boldsymbol{S}_{D D}^{*}(s) \boldsymbol{G}_{D C}^{*}(s), \\
\int_{\tau}^{\infty} e^{-s t} \exp \left(\boldsymbol{Q}_{D D} t\right) \boldsymbol{Q}_{D C} d t=\exp \left(-\left(s \boldsymbol{I}-\boldsymbol{Q}_{D D}\right) \tau\right)\left(s \boldsymbol{I}-\boldsymbol{Q}_{D D}\right)^{-1} \boldsymbol{Q}_{D C}=\boldsymbol{L}_{D D}^{*}(s) \boldsymbol{G}_{D C}^{*}(s),
\end{gathered}
$$

where

$$
\begin{gathered}
\boldsymbol{L}_{D D}^{*}(s)=\exp \left(-\left(s \boldsymbol{I}-\boldsymbol{Q}_{D D}\right) \tau\right), \\
\boldsymbol{S}_{D D}^{*}(s)=\boldsymbol{I}-\exp \left(-\left(s \boldsymbol{I}-\boldsymbol{Q}_{D D}\right) \tau\right) .
\end{gathered}
$$

In order to get more indexes and distributions for the system, we need to make some change on the system states. Considering the changeability of the states in $C$, in the following we duplicate the changeable states. More specifically, according to the assumptions in Section 2.2, the states in $C$ denoted as $C_{U}$ are changeable-up states. Then we duplicate the states in $C_{U}$ to another subset, standing for the states in $C$ when they are changeable-down states, $C_{D}$ states.

For the state space of the system, we can analyze it via the disaggregation procedure by adding a subset of states. We assume $U=\left\{1,2, \ldots, n_{U}\right\}, C=\left\{n_{U}+1, n_{U}+2, \ldots, n_{U}+n_{C}\right\}$, and $D=\left\{n_{U}+n_{C}+1, n_{U}+n_{C}+2, \ldots, n\right\}$. 
Thus, a new stochastic process $\{X(t), t \geq 0\}$ generated from duplicating a subset of state space of $\{X(t), t \geq 0\}$ is obtained. The state space of $\{X(t), t \geq 0\}$, denoted as $S$, has $n$ $\left(n=n+n_{C}\right)$ different states and $S=U \cup C_{U} \cup D \cup C_{D}$.

\subsection{Transition rules among states for the system}

The transitions among states in $S$ are shown in Figure 4 as follows.

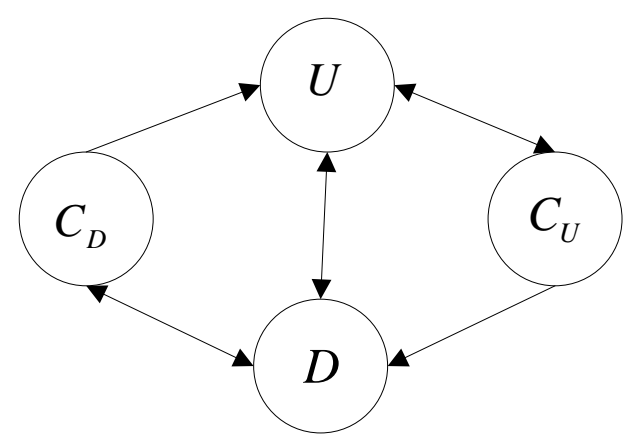

Figure 4. The transition diagram for the new Markov process

According to the assumptions of the model, $D$ can be decomposed into two parts: $D_{S}$ and $D_{L} . D_{S}$ stands for short (less than $\tau$ ) sojourns in $D$ and $D_{L}$ represents long (greater than $\tau$ ) sojourns in $D$. Then we can get a decomposed trasition diagram in Figure 5.

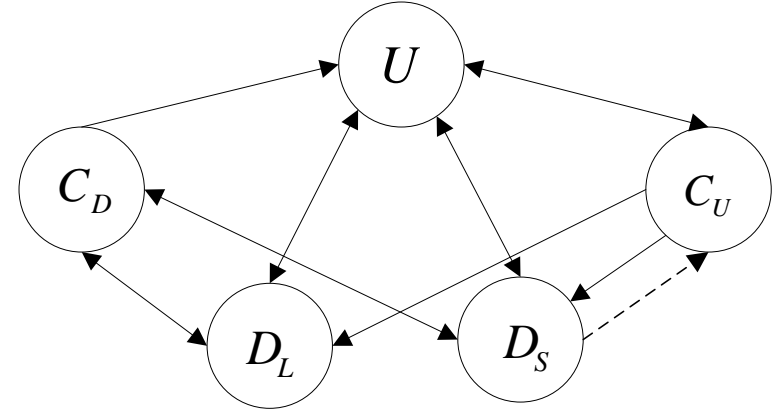

Figure 5. The decomposed transition diagram for the new system

Note that the dashed line in the transition diagram presents the difference between our new model and the original model.

The new stochastic process $\{X(t), t \geq 0\}$ is still a Markov process. However, it no longer obeys Markov properties when the neglected failure is taken into consideration. We just want to illustrate the possible transitions among the states if we need to distinguish whether the duration in $D$ is longer than $\tau$ by Figure 5, which makes the transitions rules more clearly. The transition rules are as follows: 
a) Rules for $U$ states: The bidirectional transitions can happen between $U$ and other states except $C_{D}$. Because if the system is in $U$ states following by a changeable state, then it must be a changeable-up state. Therefore, there is no transition from $U$ to $C_{D}$.

b) Rules for $D$ states shorter than $\tau$ : The bidirectional transitions can happen between $D_{S}$ and other states except $D_{L}$. What should be mentioned here is the special transition from $D_{S}$ to $C_{D}$. It happens when the state before $D_{S}$ is a Down state, then this $D_{S}$ is still regarded as in Down period. Consequently, the following changeable state is supposed to be a Down state, which is $C_{D}$.

In addition, there are no transitions between $D_{S}$ and $D_{L}$.

c) Rules for $D$ states longer than $\tau$ : The transition rules of $D_{L}$ are similar as that of $D_{S}$. It is impossible that the system runs from $D_{L}$ to $C_{U}$ and $D_{S}$.

d) Rules for changeable states $C$ : The transitions can occur between changeable states and other states, except that transitions from $U$ to $C_{D}$, from $D_{L}$ to $C_{U}$, and transitions between $C_{U}$ and $C_{D}$ are not allowed.

Then based on the new Markov process $\{X(t), t \geq 0\}$, a new perspective to analyze the system in view of the state space as $S$ of $\{X(t), t \geq 0\}$, we define that $A=U \cup C_{U}$. Thus, the system can be considered available during states set $A$. And we regard the system are "good" in states set $A$, some changeable states and short-failure states.

The properties of this system may be described by considering a semi-Markov process embedded at the time points which form the start of an available state, the start of a changeable-down sojourn, the start of a sojourn in $D$ states. Denote the semi-Markov process as $\{Z(t), t \geq 0\}$, so that $\{Z(t), t \geq 0\}$ is an aggregated stochastic process.

The semi-Markov process $\{Z(t), t \geq 0\}$ has a density kernel, which will be denoted by

$$
\boldsymbol{G}(t)=\left(\begin{array}{ccc}
\boldsymbol{0} & \boldsymbol{G}_{A D}(t) & \boldsymbol{0} \\
\boldsymbol{G}_{D A}(t) & \boldsymbol{0} & \boldsymbol{G}_{D C_{D}}(t) \\
\boldsymbol{G}_{C_{D} A}(t) & \boldsymbol{G}_{C_{D} D}(t) & \boldsymbol{0}
\end{array}\right) .
$$

For some purposes it is useful to have their Laplace transforms: 


$$
\boldsymbol{G}^{*}(s)=\left(\begin{array}{ccc}
\boldsymbol{0} & \boldsymbol{G}_{A D}^{*}(s) & \boldsymbol{0} \\
\boldsymbol{G}_{D A}^{*}(s) & \boldsymbol{0} & \boldsymbol{G}_{D C_{D}}^{*}(s) \\
\boldsymbol{G}_{C_{D} A}^{*}(s) & \boldsymbol{G}_{C_{D} D}^{*}(s) & \boldsymbol{0}
\end{array}\right) .
$$

If we ignore the durations of the sojourns and merely consider the sequence of states that are occupied at the start of each sojourn, we have an embedded Markov chain with transition probability matrix,

$$
\boldsymbol{G}=\left(\begin{array}{ccc}
\boldsymbol{0} & \boldsymbol{G}_{A D} & \boldsymbol{0} \\
\boldsymbol{G}_{D A} & \boldsymbol{0} & \boldsymbol{G}_{D C_{D}} \\
\boldsymbol{G}_{C_{D} A} & \boldsymbol{G}_{C_{D} D} & \boldsymbol{0}
\end{array}\right)=\int_{0}^{\infty} \boldsymbol{G}(t) d t=\boldsymbol{G}^{*}(0)
$$

Here we use the convention of dropping the star and the argument to denote a Laplace transform evaluated at $s=0$, which is the integral of the original function over the interval $(0, \infty)$.

\section{Reliability indexes of MRS-HDS with neglected failures}

In this section, we use the model developed in Section 3 to derive some reliability-related indexes for MRS-HDS with neglected failures, i.e., the instantaneous availability, steady-state availability and some sojourn time distributions.

\subsection{Instantaneous availability for MRS-HDS with neglected failures}

According to the definition of availability, we define the availability for a system in traditional sense, which is based on the original model. If the system is in $U$ and up-changeable states, it is regarded available for the original model. However, taking the neglected failure into consideration, the definition of the availability will be extended.

According to our new model, the system is regarded as available in Up period under several situations. Of course, the system is still available when it is in $U$ and up-changeable states. Besides, when the system is in $D$ whose duration is less than the critical value and the state the $D$ moving from is an $A$ state, then the system is still available. In addition, if the system is in $C$ state whose previous state is one short failure duration with an $A$ previous state, then it is in Up period.

Therefore, we list all the scenarios that the system is available as follows.

(1) Scenario 1: The system is in $U$ states

Apparently, the new system is available when it is in $U$ states, which is shown in Figure $6(1)$. 


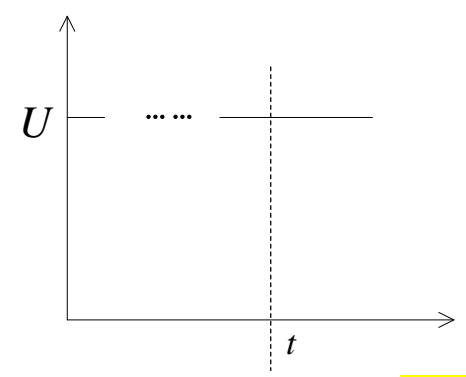

Figure 6(1). The system in $U$ states in Scenario 1

We can get the availability in this situation easily,

$$
A^{1}(t)=\pi_{0} e^{\underline{Q} t} \boldsymbol{1}_{U}
$$

where $\boldsymbol{1}_{U}=\underset{|U|}{(1, \ldots, 1,0, \ldots, 0)_{1 \times|S|}^{T} .}$

(2) Scenario 2: The system is in $C_{U}$ states

According to the description for the system, the changeable states are history dependent, so that the system is available when it is in $C_{U}$ states, which is depicted in Figure 6(2).

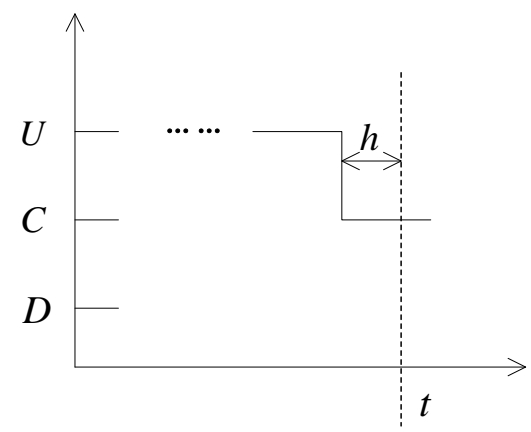

Figure 6(2). The system in $C_{U}$ states in Scenario 2

We denote the avaliability in $C_{U}$ states as $A^{2}(t)$, then

$$
A^{2}(t)=\int_{0}^{t} \pi_{0} e^{\boldsymbol{Q}(t-h)} \boldsymbol{E}_{U} \boldsymbol{Q}_{U C} e^{\boldsymbol{Q}_{C C} h} \boldsymbol{1}_{C} d h,
$$

where $\boldsymbol{E}_{U}=\left(\begin{array}{l}\boldsymbol{I}_{|U| \times|U|} \\ \boldsymbol{0}\end{array}\right)_{|S| \times|U|}, \boldsymbol{1}_{C}=(1, \ldots, 1)_{1 \times|C|}^{T}$. Note that $h$ in Figure 6(2) means the duration of the system staying in $C$ before time $t$.

The explanation of equation (12) is because the system is working at time $t-h$, then making a transition from the set $U$ to set $C$ and staying in $C$, which is called $C_{U}$, during the time length $h . \boldsymbol{1}_{C}$ is a column vector of $|C|$ ones to sum up all the desired probabilities. 
(3) Scenario 3: The system is in $D_{S}$ states

Since if the repair time in $D$ is sufficiently short, then when the failure can be neglected the system is regarded as available. In order to present the availability function, denoted as $A^{3}(t)$, we must figure out what the state before this short $D$ state is. Thus, three cases for Scenario 3 will be considered as follows.

a) Case 1 for Scenario 3: The previous state to $D_{S}$ is a $U$ state

We describe this situation by Figure 6(3a), in which the duration in $D$ is less than critical value $\tau$ (i.e. the system is in $D_{S}$ ) and the state before $D$ is $U$.

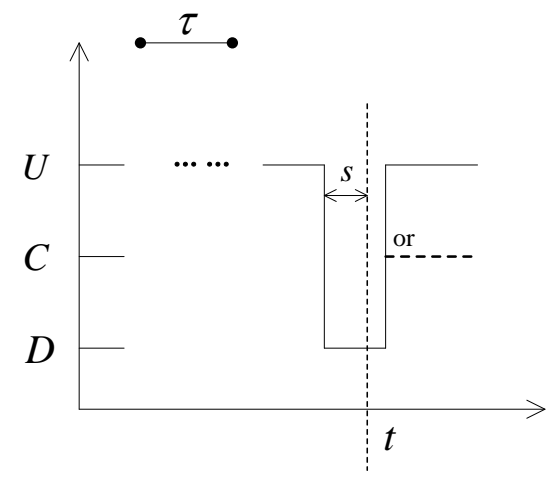

Figure 6(3a). The previous state to $D_{S}$ is a $U$ state in Scenario 3

According to the description of this situation, we can obtain the availabilty,

$$
A^{3 a}(t)=\int_{0}^{t \wedge \tau} \pi_{0} e^{\boldsymbol{Q}(t-s)} \boldsymbol{E}_{U} \boldsymbol{Q}_{U D}\left(e^{\boldsymbol{Q}_{D D} s}-e^{\boldsymbol{Q}_{D D} \tau}\right) \boldsymbol{1}_{D} d s,
$$

where $\boldsymbol{1}_{D}=(1, \ldots, 1)_{1 \times|D|}^{T}$. Note that $s$ in Figure 6.(3a) means the duration of the system staying in $D_{S}$ before time $t$.

For equation (13), because $\pi_{0} e^{\boldsymbol{Q ( t - s )}} \boldsymbol{E}_{U}$ is a probability vector which gives the probability of the repairable system being in each $U$ state at instant time $t-s$, and $\boldsymbol{Q}_{U D}$ means the system transfer from states $U$ to $D . e^{Q_{D D^{s}}}-e^{Q_{D D} \tau}$ keeps the system stays in $D_{S}$ state which means the duration in $D$ is shorter than $\tau$.

b) Case 2 for Scenario 3: The previous state to $D_{S}$ is a $C_{U}$ state

If the states before $D_{S}$ is a $C_{U}$ state, it is depicted in Figure 6.(3b). 


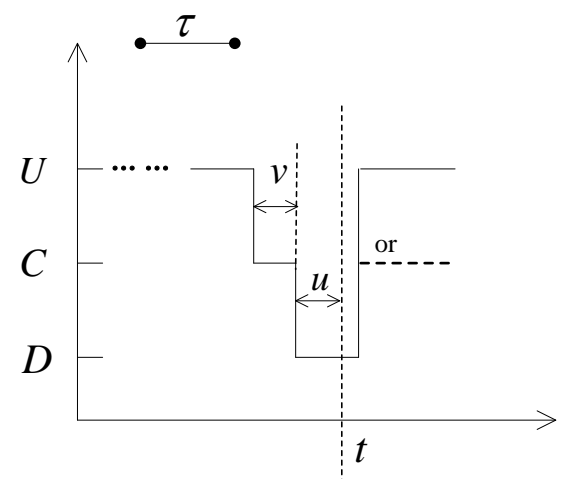

Figure 6(3b). The previous state to $D_{S}$ is a $C_{U}$ state in Scenario 3

The availability under this situation is

$$
A^{3 b}(t)=\int_{0}^{t \wedge \tau}\left[\int_{0}^{t-u} \pi_{0} e^{\boldsymbol{Q}(t-u-v)} \boldsymbol{E}_{U} \boldsymbol{Q}_{U C} e^{\boldsymbol{Q}_{C C} v} \boldsymbol{Q}_{C D}\left(e^{\boldsymbol{Q}_{D D} u}-e^{\boldsymbol{Q}_{D D} \tau}\right) \boldsymbol{I}_{D} d v\right] d u
$$

Note that in Figure 6.(3b) $u$ stands for the duration of the system staying in $D_{S}$ before time $t$ and $v$ means the duration of the system staying in $C_{U}$.

The explanation of equation (14) is: The durations that the system stays in $C_{U}$ is $v$, in $D_{S}$ until time $t$ is $u$. The system has a transition from $U$ to $C$ at time $t-u-v$ and then transfers to $D_{S}$ after $C$. Moreover, the duration in $D_{S}$ is shorter than $\tau$.

c) Case 3 for Scenario 3: The previous state to $D_{S}$ is an available $C_{D}$ state

In this case, an available $C_{D}$ state refers to that the changeable-down state with a previous $D_{S}$ state and the $D_{S}$ state has been treated in Up period. Hence, we call this kind of $C_{D}$ state as available $C_{D}$ state.

Thus, we get a transition chain that the system currently stays in an available in $D_{S}$ state with a previous available $C_{D}$ state and the available $C_{D}$ state comes from another available $D_{S}$ state. Furthermore, the second previous $D_{S}$ state could come from another available $C_{D}$ with a previous $D_{S}$ state, and so forth in the way $D_{S}$ to conditional $C_{D}$ to $D_{S}$ state.

If we denote the current $D_{S}$ state the system is staying as the $k$ th $D_{S}$ state and all the $D_{S}$ states are numbered in order as time going, then what we concern is the previous state to the 
first $D_{S}$ state. The previous state to the first $D_{S}$ state could be $U$ or $C_{U}$ states so that the availability for this case, denoted as $A^{3 c}(t)$, is again divided into two subcases. In addition, $k$ could be 2 to infinity.

- Subcase 1 of case 3 for Scenario 3: the previous state to the first $D_{S}$ state is $U$, denoted as $A^{3 c(1)}(t)$, which is depicted as figure $6(3 \mathrm{c})-1$.

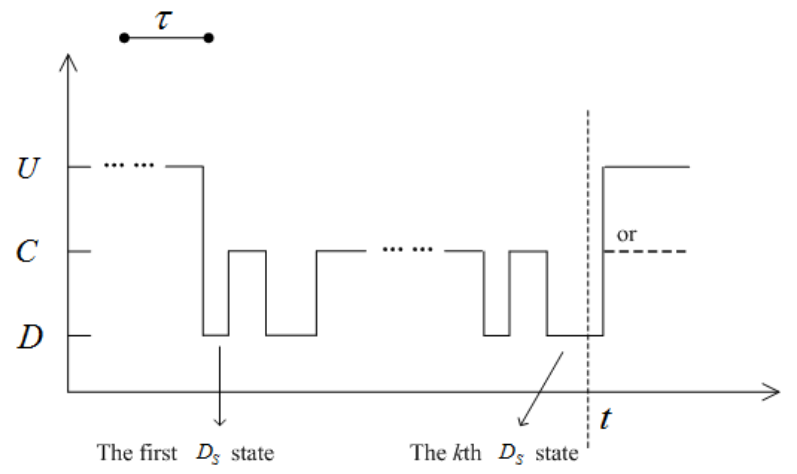

Figure 6.(3c)-1. The previous state to the first $D_{S}$ state is $U$ in Subcase 1 of case 3

$$
\begin{aligned}
& \text { for Scenario } 3 \\
& A^{3 c(1)}(t)=\sum_{k=2}^{\infty} \underbrace{\int \cdots \int}_{2 k-1} \pi_{0} e^{\boldsymbol{Q}\left(t-\sum_{i=1}^{k} u_{i}-\sum_{i=1}^{k-1} v_{i}\right)} \boldsymbol{E}_{U} \boldsymbol{Q}_{U D}\left(e^{\boldsymbol{Q}_{D D} u_{k}}-e^{\boldsymbol{Q}_{D D} \tau}\right) . \\
& {\left[\boldsymbol{Q}_{D C} e^{\boldsymbol{Q}_{C C} v_{k-1}} \boldsymbol{Q}_{C D}\left(e^{\boldsymbol{Q}_{D D} u_{k-1}}-e^{\boldsymbol{Q}_{D D} \tau}\right)\right] .} \\
& \cdots\left[\boldsymbol{Q}_{D C} e^{\boldsymbol{Q}_{C C} v_{1}} \boldsymbol{Q}_{C D}\left(e^{\boldsymbol{Q}_{D D} u_{1}}-e^{\boldsymbol{Q}_{D D} \tau}\right)\right] \boldsymbol{1}_{D} d u_{k} d v_{k-1} d u_{k-1} \cdots d v_{1} d u_{1} \text {. }
\end{aligned}
$$

- Subcase 2 of case 3 for Scenario 3: the previous state to the first $D_{S}$ state is $C_{U}$, denoted as $A^{3 c(2)}(t)$, which is depicted as figure $6(3 \mathrm{c})-2$. 


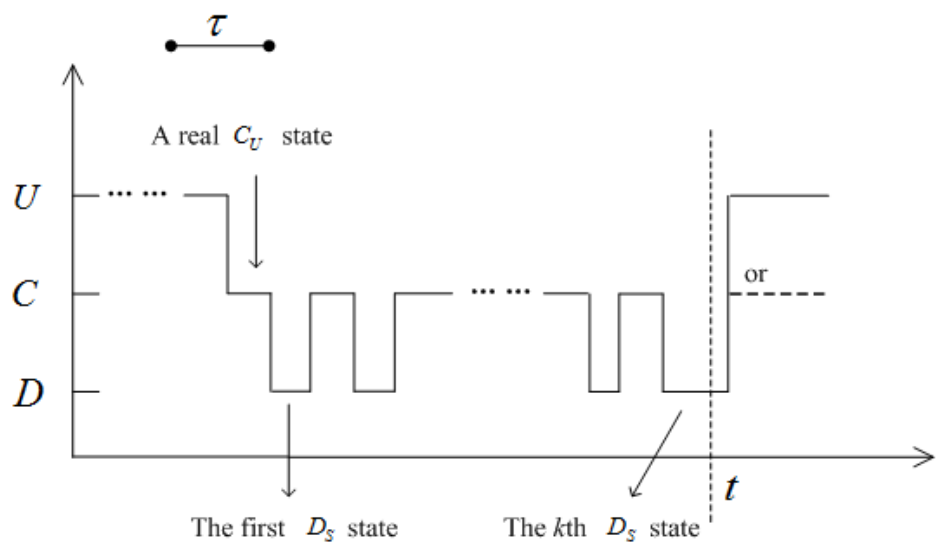

Figure 6.(3c)-2. The previous state to the first $D_{S}$ state is $C_{U}$ in Subcase 2 of case 3

$$
\begin{aligned}
& \text { for Scenario } 3 \\
& A^{3 c(2)}(t)=\sum_{k=2}^{\infty} \underbrace{\int \cdots \int}_{2 k} \pi_{0} e^{\boldsymbol{Q}\left(t-\sum_{i=1}^{k} u_{i}-\sum_{i=1}^{k} v_{i}\right)} \boldsymbol{E}_{U} \boldsymbol{Q}_{U C} e^{\boldsymbol{Q}_{C C} v_{k}} \boldsymbol{Q}_{C D}\left(e^{\boldsymbol{Q}_{D D} u_{k}}-e^{\boldsymbol{Q}_{D D} \tau}\right) \\
& {\left[\boldsymbol{Q}_{D C} e^{\boldsymbol{Q}_{C C} v_{k-1}} \boldsymbol{Q}_{C D}\left(e^{\boldsymbol{Q}_{D D} u_{k-1}}-e^{\boldsymbol{Q}_{D D} \tau}\right)\right] .} \\
& \cdots\left[\boldsymbol{Q}_{D C} e^{\boldsymbol{Q}_{C C} v_{1}} \boldsymbol{Q}_{C D}\left(e^{\boldsymbol{Q}_{D D} u_{1}}-e^{\boldsymbol{Q}_{D D} \tau}\right)\right] \boldsymbol{1}_{D} d v_{k} d u_{k} d v_{k-1} d u_{k-1} \cdots d v_{1} d u_{1} \text {. }
\end{aligned}
$$

And,

$$
A^{3 c}(t)=A^{3 c(1)}(t)+A^{3 c(2)}(t) .
$$

Thus,

$$
A^{3}(t)=A^{3 a}(t)+A^{3 b}(t)+A^{3 c}(t) .
$$

(4) Scenario 4: The system is in an available $C_{D}$ state

Basically, the system is not available in $C_{D}$ states in the original Markov history-dependent model. However, the system can be regarded as available when it is in an available $C_{D}$ state with a previous $D_{S}$ state. Similar with Scenario 3, the availability of this scenario denoted as $A^{4}(t)$, depends on what the state is before the previous $D_{S}$ state. Also, three cases for Scenario 4 will be considered as follows.

a) Case 1 for Scenario 4: The previous state to $D_{S}$ is a $U$ state 


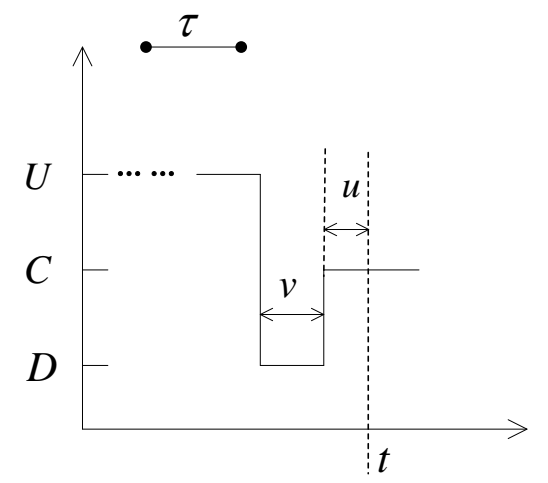

Figure 6.(4a). The previous state to $D_{S}$ is a $U$ state in Scenario 4

According to the analysis for this situation, if the previous state to $D_{S}$ is a $U$ state, we can get the availability $A^{4 a}(t)$ as follows. This situation is illustrated in Figure 6(4a).

$$
A^{4 a}(t)=\int_{0}^{t}\left[\int_{0}^{(t-u) \wedge \tau} \pi_{0} e^{\boldsymbol{Q}(t-u-v)} \boldsymbol{E}_{U} \boldsymbol{Q}_{U D} e^{\boldsymbol{Q}_{D D} v} \boldsymbol{Q}_{D C} e^{\boldsymbol{Q}_{C C^{u}}} \boldsymbol{1}_{C} d v\right] d u
$$

Note that in Figure 6.(4a) $u$ stands for the duration of the system staying in $C_{D}$ before time $t$ and $v$ means the duration of the system staying in $D_{S}$.

For equation (19), it is because that the system has a transition from $U$ to $D$ at time $t-u-v$ and then spends $v$ stays at $D$ ( $v$ is shorter than $\tau$ ). Then, the system transfers from $D_{S}$ to $C$ (it is $C_{D}$ ) and the duration in $C_{D}$ until time $t$ is $u$.

b) Case 2 for Scenario 4: The previous state to $D_{S}$ is a $C_{U}$ state

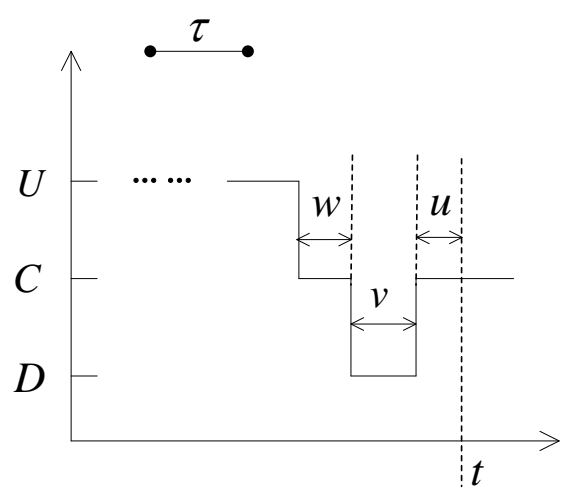

Figure 6.(4b). The previous state to $D_{S}$ is a $C_{U}$ state in Scenario 4

If the previous state to $D_{S}$ is a $C_{U}$ state, the situation is shown in Figure 6(4b). Note that in Figure 6.(4b) $u$ stands for the duration of the system staying in $C_{D}$ before time $t, v$ 
means the duration of the system staying in $D_{S}$ and $w$ represents the duration of the system staying in $C_{U}$.

Similarly, we can obtain the $A^{4 b}(t)$ is

$$
A^{4 b}(t)=\int_{0}^{t}\left\{\int_{0}^{(t-u) \wedge \tau}\left[\int_{0}^{t-u-v} \pi_{0} e^{\boldsymbol{Q}(t-u-v-w)} \boldsymbol{E}_{U} \boldsymbol{Q}_{U C} \boldsymbol{Q}^{\boldsymbol{Q}_{C C} w} \boldsymbol{Q}_{C D} e^{\boldsymbol{Q}_{D D} v} \boldsymbol{Q}_{D C} e^{\boldsymbol{Q}_{C C} u} \boldsymbol{1}_{C} d w\right] d v\right\} d u
$$

The explanation of equation (20) is similar to that of equation (16), adding the consideration of the system has a transition to $C_{U}$ after $U$ and before $D_{S}$.

c) Case 3 for Scenario 4: The previous state to $D_{S}$ is an available $C_{D}$ state

The availability for this case, denoted as $A^{4 c}(t)$, is handled similarly with $A^{3 c}(t)$. It is again divided into two subcases, the explanation can be reffered to that in Case 3 for Scenario 3 .

- Subcase 1 of case 3 for Scenario 4: The previous state to the first $D_{S}$ state is $U$,

denoted as $A^{4 c(1)}(t)$, which is depicted as figure $6(4 \mathrm{c})-1$.

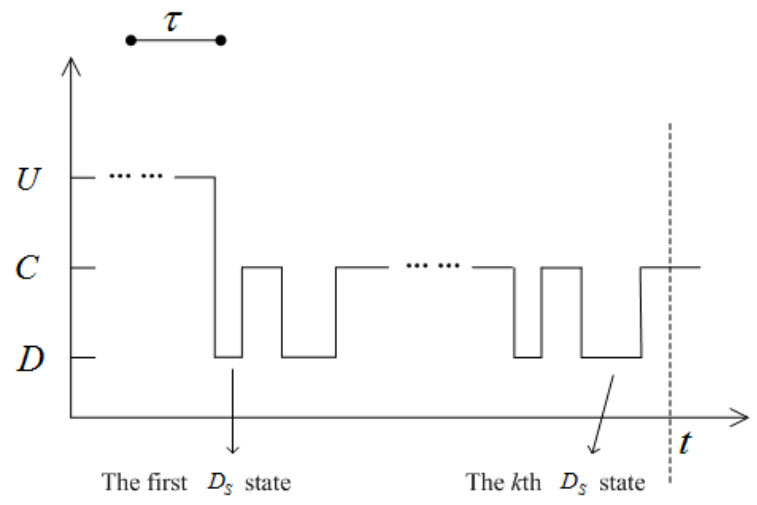

Figure 6.(4c)-1. The previous state to the first $D_{S}$ state is $U$ in

Subcase 1 of case 3 for Scenario 4

$$
\begin{aligned}
A^{4 c(1)}(t)= & \sum_{k=2}^{\infty} \underbrace{\int \cdots \int}_{2 k} \pi_{0} e^{\boldsymbol{Q}\left(t-\sum_{i=1}^{k} u_{i}-\sum_{i=1}^{k} v_{i}\right)} \boldsymbol{E}_{U} \boldsymbol{Q}_{U D} e^{\boldsymbol{Q}_{D D} u_{k}} \boldsymbol{Q}_{D C} e^{\boldsymbol{Q}_{C C} v_{k}} \\
& {\left[\boldsymbol{Q}_{C D} e^{\boldsymbol{Q}_{D D} u_{k-1}} \boldsymbol{Q}_{D C} e^{\boldsymbol{Q}_{C C} v_{k-1}}\right] \cdot } \\
& \cdots\left[\boldsymbol{Q}_{C D} e^{\boldsymbol{Q}_{D D} u_{1}} \boldsymbol{Q}_{D C} e^{\boldsymbol{Q}_{C C} v_{1}}\right] \boldsymbol{I}_{C} d u_{k} d v_{k} d u_{k-1} d v_{k-1} \cdots d u_{1} d v_{1} .
\end{aligned}
$$

- Subcase 2 of case 3: The previous state to the first $D_{S}$ state is $C_{U}$, denoted as $A^{4 c(2)}(t)$, which is depicted as Figure $6(4 c)-2$. 


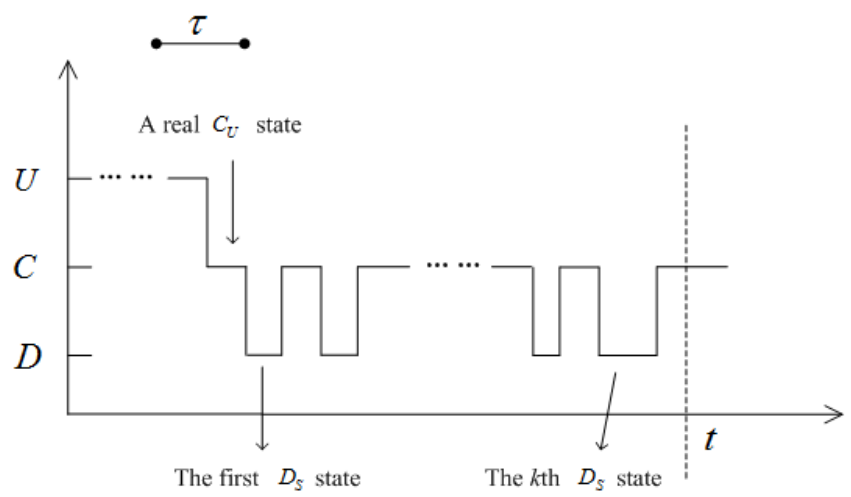

Figure 6.(4c)-2. The previous state to the first $D_{S}$ state is real $C_{U}$ in

Subcase 1 of case 3 for Scenario 4

$$
\begin{aligned}
A^{4 c(2)}(t)= & \sum_{k=2}^{\infty} \underbrace{\int \cdots \int}_{2 k+1} \pi_{0} e^{\boldsymbol{Q}\left(t-\sum_{i=1}^{k} u_{i}-\sum_{i=1}^{k+1} v_{i}\right)} \boldsymbol{E}_{U} \boldsymbol{Q}_{U C} e^{\boldsymbol{Q}_{C C} v_{k+1}} \\
& \boldsymbol{Q}_{C D} e^{\boldsymbol{Q}_{D D} u_{k}} \boldsymbol{Q}_{D C} e^{\boldsymbol{Q}_{C C} v_{k}}\left[\boldsymbol{Q}_{C D} e^{\boldsymbol{Q}_{D D} u_{k-1}} \boldsymbol{Q}_{D C} e^{\boldsymbol{Q}_{C C} v_{k-1}}\right] \cdot \\
& \cdots\left[\boldsymbol{Q}_{C D} e^{\boldsymbol{Q}_{D D} u_{1}} \boldsymbol{Q}_{D C} e^{\boldsymbol{Q}_{C C} v_{1}}\right] \boldsymbol{1}_{C} d v_{k+1} d u_{k} d v_{k} d u_{k-1} d v_{k-1} \cdots d u_{1} d v_{1} .
\end{aligned}
$$

Theoretically,

$$
A^{4 c}(t)=A^{4 c(1)}(t)+A^{4 c(2)}(t) .
$$

Similar with that in Case 3 for Scenario 3, all the expressions for $A^{4 c}(t)$ will turn to be tiny when computation is done, which will be demonstrated in the numerical example.

Thus,

$$
A^{4}(t)=A^{4 a}(t)+A^{4 b}(t)+A^{4 c}(t) .
$$

Finally, we can get the instantaneous availability for the system as follows,

$$
A(t)=A^{1}(t)+A^{2}(t)+A^{3}(t)+A^{4}(t),
$$

and the matrices in this formula have been already obtained in the previous section.

\subsection{Steady-state availability for MRS-HDS with neglected failures}

According to the definition, the steady-state availability can be got from the limitation of instantaneous availability, when time $t$ approaches infinity.

We denote the steady-state availability as $A(\infty)$, and

$$
A(\infty)=\lim _{t \rightarrow \infty} A(t) .
$$

Therefore, after we derive the function for instantaneous availability, we take the limit to get the steady-state availability for the system. It will be shown in the numerical example section later. 


\subsection{Some sojourn time distributions for MRS-HDS with neglected failures}

Based on the analysis in Section 3.1, we can present the transition matrix $\boldsymbol{Q}$ for the new Markov process $\{X(t), t \geq 0\}$,

$$
\boldsymbol{Q}=\left(\begin{array}{cccc}
\boldsymbol{Q}_{U U} & \boldsymbol{Q}_{U C_{U}} & \boldsymbol{0} & \boldsymbol{Q}_{U D} \\
\boldsymbol{Q}_{C_{U} U} & \boldsymbol{Q}_{C_{U} C_{U}} & \boldsymbol{0} & \boldsymbol{Q}_{C_{U} D} \\
\boldsymbol{Q}_{C_{D} U} & \boldsymbol{0} & \boldsymbol{Q}_{C_{D} C_{D}} & \boldsymbol{Q}_{C_{D} D} \\
\boldsymbol{Q}_{D U} & \boldsymbol{0} & \boldsymbol{Q}_{D C_{D}} & \boldsymbol{Q}_{D D}
\end{array}\right)
$$

Since we have defined that $A=U \cup C_{U}$, from matrix $Q$ we can get

$$
\boldsymbol{Q}_{A A}=\left(\begin{array}{cc}
\boldsymbol{Q}_{U U} & \boldsymbol{Q}_{U C_{U}} \\
\boldsymbol{Q}_{C_{U} U} & \boldsymbol{Q}_{C_{U} C_{U}}
\end{array}\right), \boldsymbol{Q}_{A D}=\left(\begin{array}{c}
\boldsymbol{Q}_{U D} \\
\boldsymbol{Q}_{C_{U} D}
\end{array}\right), \boldsymbol{Q}_{D A}=\left(\begin{array}{ll}
\boldsymbol{Q}_{D U} & \boldsymbol{Q}_{D C_{U}}
\end{array}\right), \boldsymbol{Q}_{D D}=\boldsymbol{Q}_{D D}
$$

And

$$
\begin{aligned}
& \boldsymbol{G}_{A D}^{*}(s)=\left(s \boldsymbol{I}-\boldsymbol{Q}_{A A}\right)^{-1} \boldsymbol{Q}_{A D}, \\
& \boldsymbol{G}_{D A}^{*}(s)=\left(s \boldsymbol{I}-\boldsymbol{Q}_{D D}\right)^{-1} \boldsymbol{Q}_{D A} .
\end{aligned}
$$

According to what has been discussed in Section 3.1 about Up and Down periods for the system, combining with the meanings of the semi-Markov process, the elements in the density kernel ${ }^{e} \overline{\boldsymbol{G}}(t)$ (in equation (3)) can be obtained.

Firstly, the Laplace transform of ${ }^{e} \overline{\boldsymbol{G}}_{W F}(t)$ is given by the following equation,

$$
{ }^{e} \overline{\boldsymbol{G}}_{W F}^{*}(s)=G 0+G 1+G 2+G 3+G 4+G 5+G 6,
$$

where

$$
\begin{aligned}
& G 0=\boldsymbol{G}_{U C}^{*}(s) \boldsymbol{G}_{C D}^{*}(s) L_{D D}^{*}(0), \\
& G 1=\sum_{r=0}^{\infty}\left[\boldsymbol{G}_{U C}^{*}(s) \boldsymbol{G}_{C U}^{*}(s)\right]^{r}\left[\boldsymbol{G}_{U C}^{*}(s) \boldsymbol{G}_{C D}^{*}(s) L_{D D}^{*}(0)+\boldsymbol{G}_{U D}^{*}(s) L_{D D}^{*}(0)\right], \\
& G 2=\sum_{r=0}^{\infty}\left[\boldsymbol{G}_{U D}^{*}(s) S_{D D}^{*}(s) \boldsymbol{G}_{D U}^{*}(s)\right]^{r}\left[\boldsymbol{G}_{U C}^{*}(s) \boldsymbol{G}_{C D}^{*}(s) L_{D D}^{*}(0)+\boldsymbol{G}_{U D}^{*}(s) L_{D D}^{*}(0)\right], \\
& G 3=\boldsymbol{G}_{U C}^{*}(s) \sum_{r=0}^{\infty}\left[\boldsymbol{G}_{C D}^{*}(s) S_{D D}^{*}(s) \boldsymbol{G}_{D C}^{*}(s)\right]^{r} \\
& \cdot\left\{\boldsymbol{G}_{C U}^{*}(s)\left[\boldsymbol{G}_{U C}^{*}(s) \boldsymbol{G}_{C D}^{*}(s) L_{D D}^{*}(0)+\boldsymbol{G}_{U D}^{*}(s) L_{D D}^{*}(0)\right]+\boldsymbol{G}_{C D}^{*}(s) L_{D D}^{*}(0)\right\},
\end{aligned}
$$




$$
\begin{aligned}
& G 4=\sum_{r=0}^{\infty}\left[\boldsymbol{G}_{U C}^{*}(s) \boldsymbol{G}_{C D}^{*}(s) S_{D D}^{*}(s) \boldsymbol{G}_{D C}^{*}(s) \boldsymbol{G}_{C U}^{*}(s)\right]^{r} \\
& \cdot\left[\boldsymbol{G}_{U C}^{*}(s) \boldsymbol{G}_{C D}^{*}(s) L_{D D}^{*}(0)+\boldsymbol{G}_{U D}^{*}(s) L_{D D}^{*}(0)\right], \\
& G 5=\sum_{r=0}^{\infty}\left[\boldsymbol{G}_{U D}^{*}(s) S_{D D}^{*}(s) \boldsymbol{G}_{D C}^{*}(s) \boldsymbol{G}_{C U}^{*}(s)\right]^{r}\left[\boldsymbol{G}_{U C}^{*}(s) \boldsymbol{G}_{C D}^{*}(s) L_{D D}^{*}(0)+\boldsymbol{G}_{U D}^{*}(s) L_{D D}^{*}(0)\right], \\
& G 6=\boldsymbol{G}_{U D}^{*}(s) S_{D D}^{*}(s) \boldsymbol{G}_{D C}^{*}(s) \sum_{r=0}^{\infty}\left[\boldsymbol{G}_{C D}^{*}(s) S_{D D}^{*}(s) \boldsymbol{G}_{D C}^{*}(s)\right]^{r} \\
& \cdot\left\{\boldsymbol{G}_{C U}^{*}(s)\left[\boldsymbol{G}_{U C}^{*}(s) \boldsymbol{G}_{C D}^{*}(s) L_{D D}^{*}(0)+\boldsymbol{G}_{U D}^{*}(s) L_{D D}^{*}(0)\right]+\boldsymbol{G}_{C D}^{*}(s) L_{D D}^{*}(0)\right\} .
\end{aligned}
$$

The Laplace transform of ${ }^{e} \overline{\boldsymbol{G}}_{W F}(t)$ can be presented as follows,

$$
\begin{aligned}
& { }^{e} \overline{\boldsymbol{G}}_{F W}^{*}(s)= \\
& L_{D D}^{*}(s) \boldsymbol{G}_{D U}^{*}(s)+L_{D D}^{*}(s) \boldsymbol{G}_{D C}^{*}(s) \sum_{r=0}^{\infty}\left[\boldsymbol{G}_{C D}^{*}(s) \boldsymbol{G}_{D C}^{*}(s)\right]^{r}\left[\boldsymbol{G}_{U C}^{*}(s)+\boldsymbol{G}_{C D}^{*}(s) \boldsymbol{G}_{D U}^{*}(s)\right] .
\end{aligned}
$$

Next, some distributions for the Markov history-dependent repairable system can be obtained as follows.

(1) The distribution for the $k$ th working period from the beginning up states

We suppose the system is in up states ( $U$ states) at the beginning. Let $\pi_{W}^{(k-1)}$ be the state occupancy probability vector at the start of the $k$ th working period, $k \in\{1,2, \ldots\}$.

Then, the probability density function for the duration of the $k$ th working period is given by

$$
\pi_{W}^{(k-1)}{ }^{e} \overline{\boldsymbol{G}}_{W F}(t) \boldsymbol{1}_{F},
$$

where $\pi_{W}^{(k)}=\pi_{W}^{(k-1)}{ }^{e} \overline{\boldsymbol{G}}_{W F}(\infty){ }^{e} \overline{\boldsymbol{G}}_{F W}(\infty)$, and $\pi_{W}^{(0)}=\pi_{W}$, which can be got from the initial probability vector $\pi_{0}=\left(\pi_{W}, \pi_{F}\right) . \boldsymbol{1}_{F}$ is a column vector of $|F|$ ones.

From eqution (25), we can know the Laplace transform of ${ }^{e} \overline{\boldsymbol{G}}_{W F}(t)$.

Therefore, we can get the probability density function for the duration of the $k$ th working period by computation of inverse Laplace transform and normalazition.

(2) The distribution for the $k$ th failure period

Correspondingly, if we focus on the failure period (Down period of the system) instead of working period (Up period of the system), the probability density function for the duration of the $k$ th failure period is given by 


$$
\pi_{F}^{(k-1)}{ }^{e} \overline{\boldsymbol{G}}_{F W}(t) \boldsymbol{1}_{W},
$$

where $\pi_{F}^{(k)}=\pi_{F}^{(k-1)}{ }^{e} \overline{\boldsymbol{G}}_{F W}(\infty){ }^{e} \overline{\boldsymbol{G}}_{W F}(\infty)$, and $\pi_{F}^{(0)}=\pi_{F}$, which can be got from the initial probability vector $\pi_{0}=\left(\pi_{W}, \pi_{F}\right) . \boldsymbol{1}_{W}$ is a column vector of $|W|$ ones.

Similarly, we have got the Laplace transform of ${ }^{e} \overline{\boldsymbol{G}}_{W F}(t)$ by equation (26). Consequently, the probability density function for the duration of the $k$ th failure period can be obtained.

(3) The distribution for the real working time in an effective working period

In fact, there is some time the system is not at working as the short failure is neglected for the Markov history-dependent repairable system. Thus, we can consider the real working time in a working period.

The Laplace transform of the probability density function for the real working time in the $k$ th working period is

$$
\sum_{r=0}^{\infty} \pi_{A}^{(k-1)}\left[\boldsymbol{G}_{A D}^{*}(s) S_{D D}^{*}(0) \boldsymbol{G}_{D A}^{*}(0)\right]^{r} \boldsymbol{G}_{A D}^{*}(s) L_{D D}^{*}(0) \boldsymbol{1}_{D}
$$

Where $\pi_{A}^{(k-1)}$ can be got from the $k$ th working probability vector $\pi_{W}^{(k-1)}=\left(\pi_{A}^{(k-1)}, \pi_{W-A}^{(k-1)}\right) . \boldsymbol{1}_{D}$ is a column vector of $|D|$ ones.

Hence, the probability density function can be obtained after doing inverse Laplace transform and normalazition.

(4) The distribution for the neglected failure time in an effective working period

Since the short failure in a working period is neglected, we also concern the distribution for the neglected failure time in the $k$ th working period.

The Laplace transform of the probability density function for the neglected failure time in the $k$ th working period is

$$
\sum_{r=0}^{\infty} \pi_{A}^{(k-1)}\left[\boldsymbol{G}_{A D}^{*}(0) S_{D D}^{*}(s) \boldsymbol{G}_{D A}^{*}(s)\right]^{r} \boldsymbol{G}_{A D}^{*}(0) L_{D D}^{*}(0) \boldsymbol{1}_{D}
$$

Finally, Inverse Laplace transform and normalazition are needed to do as well.

\section{Numerical example}

A numerical example is presented in this section to demonstrate the methods developed in previous sections. For simplicity, we assume that there is only one state in each set $U, C$, and $D$. Assume the transition rate matrix for the original Markov process describing the repairable system is 


$$
Q=\left(\begin{array}{ccc}
-3 & 1 & 2 \\
4 & -6 & 2 \\
3 & 2 & -5
\end{array}\right),
$$

and we assume that $\tau=0.1$ and that the initial state of the system is an Up state, so that the initial probability vector is $\pi_{0}=(1,0,0)$.

Next, we duplicate the changeable state to yield the transition rate matrix for the aggregated Markov process $\{X(t), t \geq 0\}$ :

$$
\boldsymbol{Q}=\left(\begin{array}{cccc}
-3 & 1 & 0 & 2 \\
3 & -4 & 0 & 1 \\
1 & 0 & -2 & 1 \\
3 & 0 & 2 & -5
\end{array}\right)
$$

To calculate the instantaneous availability, we first calculate the expressions for $A^{i}(t)$, $i=1,2,3,4$, based on the equations obtained in Section 4.1. It should be noted that we only consider the first two terms in the expressions for $A^{3}(t)$ (Eq. (18)) and $A^{4}(t)$ (Eq. (24)), respectively. This is because both $A^{3 c}(t)$ and $A^{4 c}(t)$ involve the continuous and repeated transitions between specific states, whose probability, compared to other terms in (18) and (24), is negligible. The results are presented by the solid line in Figure 7. Monte Carlo simulations are conducted to verify the derived analytical expressions. We equally divide $[0,3]$ into 500 sub-intervals and use $10^{4}$ random samples to calculate the availability at each sub-interval. The results are also given in Figure 7. From the comparisons, it can be seen clearly that the derived equations accurately describe the availability of the system.

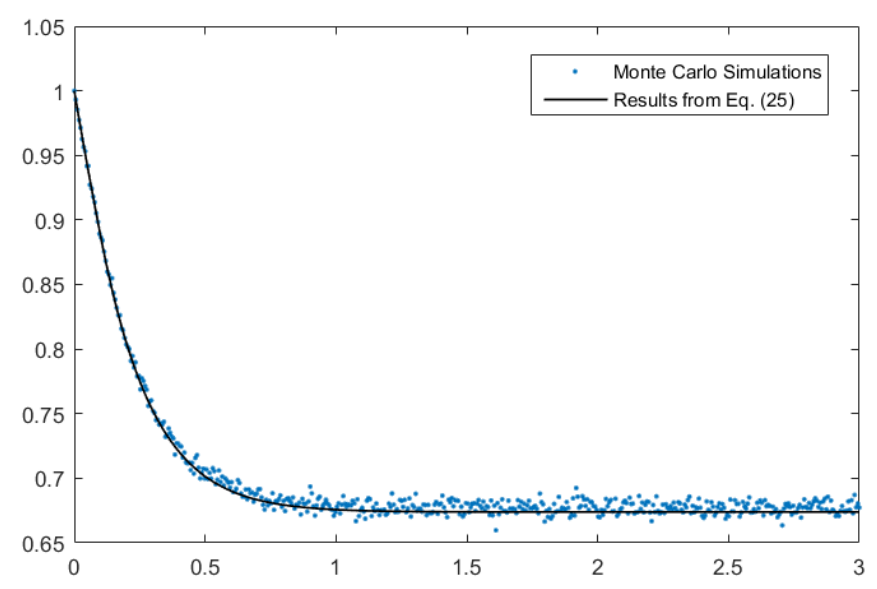

Figure 7. The instantaneous availability for the MRS-HDS with neglected failures compared with the result of simulation 
Steady-state availability can, then, be obtained through calculating:

$$
A(\infty)=0.6739 \text {. }
$$

Next, we calculate the distribution of some sojourn times. For illustration purposes, we only calculate the distribution of the real working times in an effective working period. Other distributions discussed in Section 4.3 can be achieved in a similar way. From the values of matrix

$\boldsymbol{Q}$ in equation (34), we can get matrices in equation (28),

$$
\boldsymbol{Q}_{A A}=\left(\begin{array}{cc}
-3 & 1 \\
3 & -4
\end{array}\right), \boldsymbol{Q}_{A D}=\left(\begin{array}{l}
2 \\
1
\end{array}\right), \boldsymbol{Q}_{D A}=\left(\begin{array}{ll}
3 & 0
\end{array}\right), \boldsymbol{Q}_{D D}=(-5) .
$$

Therefore, equations (28) and (29) become,

$$
\boldsymbol{G}_{A D}^{*}(s)=\left(\begin{array}{c}
\frac{2 s+9}{s^{2}+7 s+9} \\
\frac{s+9}{s^{2}+7 s+9}
\end{array}\right), \quad \boldsymbol{G}_{D A}^{*}(s)=\left(\begin{array}{cc}
\frac{3}{s+5} & 0
\end{array}\right) .
$$

Assume the predefined critical value is $\tau=1$. From equations (6) and (7), we can get the expression of $L_{D D}^{*}(s), S_{D D}^{*}(s), L_{D D}^{*}(0)$ and $S_{D D}^{*}(0)$. Based on equation (29-n33), we calculate the real working times in the first working period with $k=1, \pi_{A}^{(0)}=\left(\begin{array}{ll}1 & 0\end{array}\right)$, as shown in Figure 8.

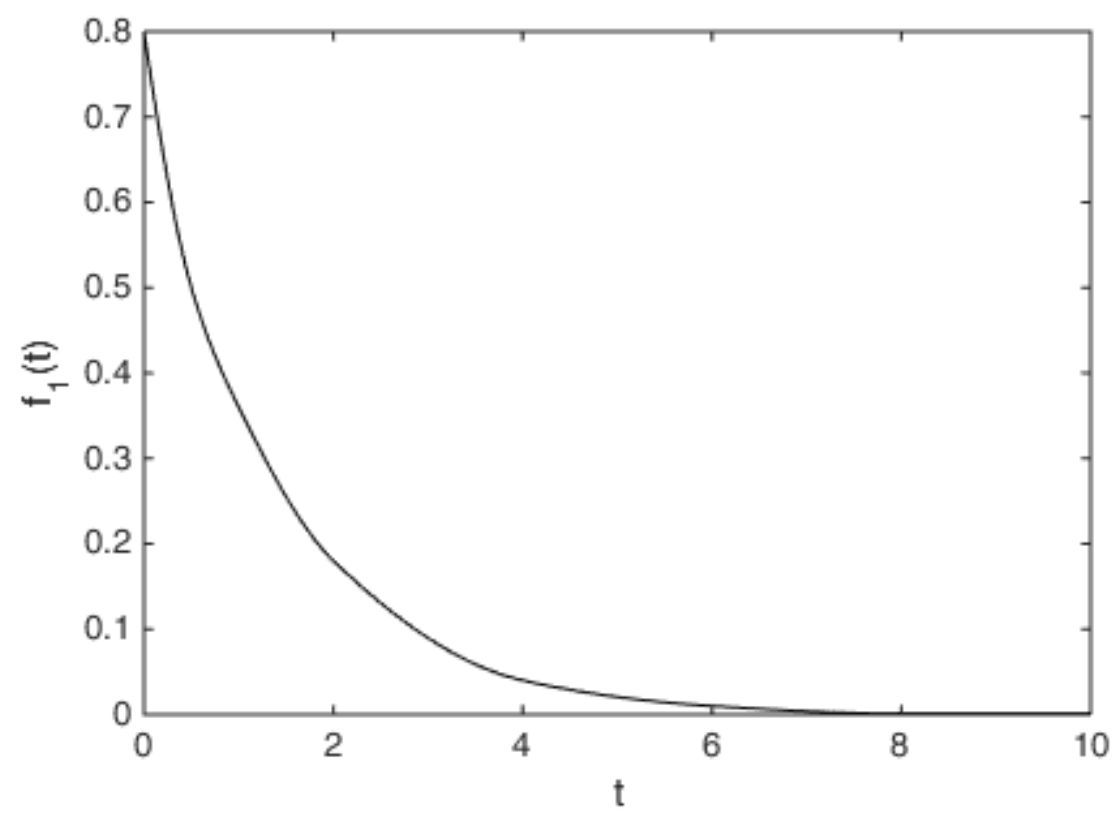

Figure 8 . The probability density function for the real working times in the first working period

\section{Conclusions}

In this paper, the theory of aggregated stochastic processes is employed to develop reliability analysis methods for Markov Repairable System with History-Dependent up and down States (MRS-HDS) considering the effect of neglected failures. A new model for MRS-HDS with 
neglected failures is developed first, based on the theory of aggregated stochastic processes, which allows for the explicit consideration of both history-dependent states and neglected failures in repairable system models. Based on the developed model, the instantaneous and steady-state availabilities of MRS-HDS with neglected failures are derived. Also, some reliability-related time distributions are obtained, including, distribution for the $k$ th working period, distribution for the $k$ th failure period, distribution for the real working time in an effective working period, distribution for the neglected failure time in an effective working period. All the derived reliability indexes can be expressed in closed-form, which provides an effective and comprehensive way to describe the reliability of MRS-HDS with neglected failures.

In the future, more versatile reliability indexes might be defined and derived so that the behavior of system reliability from different aspects could be better captured. Moreover, considering the influence of system configuration, more system structures can be investigated to achieve a more comprehensive understanding of reliability analysis of MRS-HDS, e.g., series systems, parallel systems, coherent systems and so on.

\section{Acknowledgments}

The research of Dr. Shijia Du is supported by the National Natural Science Foundation of China (No. 71601010) and China Postdoctoral Science Foundation (No. 2016M590030). The research of Dr. Zhiguo Zeng is supported by National Natural Science Foundation of China (61573041 and 71671009). The research of Professor Rui Kang is supported by National Natural Science Foundation of China (61573041).

\section{References}

[1] Liu B L, Cui L R, Wen Y Q, Shen J Y (2015) A cold standby repairable system with working vacations and vacation interruption following Markovian arrival process, Reliability Engineering and System Safety 142: 1-8.

[2] Lu J M, Wu X Y, Liu Y L, Lundteigen M A (2015) Reliability analysis of large phased-mission systems with repairable components based on success-state sampling, Reliability Engineering and System Safety 142: 123-133.

[3] Song S, Coit D W, Feng Q, Peng H (2014) Reliability analysis for multi-component systems subject to multiple dependent competing failure, IEEE Transactions on Reliability 63(1): 331-345.

[4] Montoro-Cazorla D, Rafael Pérez-Ocón R (2014) A reliability system under different types of shock governed by a Markovian arrival process and maintenance policy $\mathrm{K}$, European Journal of Operational Research 235: 636-642.

[5] Csenki A (2007) Joint interval reliability for Markov systems with an application in transmission line reliability, Reliability Engineering and System Safety 92(6): 685-696.

[6] Yu M, Cui L (2010) A study of optimal availability for a two-stage directed network of Markov repairable systems, Proceedings of the Fourth Asia-Pacific International Symposium, 796-803.

[7] Liu B L, Cui L R, Wen Y Q (2014) Interval reliability for aggregated Markov repairable system with repair time omission, Annals of Operations Research 212(1): 169-183. 
[8] Cui L R, Li H J, Li J L (2007) Markov repairable systems with history-dependent up and down states, Stochastic Models23: 665-681.

[9] Wang L, Cui L (2011) Aggregated semi-Markov repairable systems with history-dependent up and down states, Mathematical and Computer Modelling53: 883-895.

[10] Wang W G, Du S J, Cui L R (2015) Multi-Point and Multi-Interval Availabilities for Markov Repairable Systems with History-Dependent Up and Down States, Journal of Shanghai Jiaotong University (Science), 2015, 20(3): 349-352.

[11] Zheng Z H, Cui L R, Li H J (2008) Availability of semi-Markov repairable systems with history-dependent up and down states, in Proceedings of the Third Asian International Workshop, pp. 186-193.

[12] Zheng Z H, Cui L R, Hawkes A G (2006) A study on a single-unit Markov repairable system with repair time omission, IEEE Transactions on Reliability 55(2): 182-188.

[13] Bao X Z, Cui L R (2010) An analysis of availability for series Markov repairable system with neglected or delayed failures, IEEE Transactions on Reliability 59(4): 734-743.

[14] Du S, Cui L, Li H, Zhao X (2013) A study on joint availability for $k$ out of $n$ and consecutive $k$ out of $n$ points and intervals, Quality Technology and Quantitative Management 10(2): 179-191.

[15] Cui L, Du S, Liu B (2013) Multi-point and multi-interval availabilities, IEEE Transactions on Reliability 62(4): 811-820.

[16] Du S, Lin C, Cui L (2014) Reliabilities of a single-unit system with multi-phased missions, Communications in Statistics-Theory and Methods. DOI: 10.1080/03610926.2014.882953.

[17] Burke C J, Rosenblatt M (1957) A Markovian function of a Markovchain, Annals of Mathematical Statistic 29: 1112-1122.

[18] Colquhoun D, Hawkes A G (1977) Relaxation and fluctuations of membrane currents that flow through drug-operated ion channels, Philosophical Transactions of the Royal Society of London Series B-Biological Sciences 199: 231-262.

[19] Colquhoun D, Hawkes A G (1982) On the stochastic properties of bursts of single Ion Channel openings and of clusters of bursts, Philosophical Transactions of the Royal Society of London Series B-Biological Sciences 300: 1-59.

[20] Rubino G, Sericola B (1989) Sojourn times in finite Markov processes, Journal of Applied Probability 26: 744-756.

[21] Hawkes A G, Cui L, Zheng Z (2011) Modeling the evolution of system reliability performance under alternative environments, IIE Transactions 43: 761-772.

[22] Cui L, Du S, Hawkes A G (2012) A study on a single-unit repairable system with state aggregations, IIE Transactions 44: 1022-1032.

[23] Cui L, Du S, Zhang A (2014) Reliability measures for two-part partition of states for aggregated Markov repairable systems, Annals of Operations Research 212(1): 93-114.

[24] Wang L, Cui L (2013) Performance evaluation of aggregated Markov repairable systems with multi-operating levels, Asia-Pacific Journal of Operational Research 30(4) 1350003: $1-27$.

[25] Wang L Y, Jia X, Zhang J (2013) Reliability Evaluation for Multi-State Markov Repairable Systems with Redundant Dependencies, Quality Technology and Quantitative Management 10(3): 277-289. 
[26] Liu B L, Cui L R, Wen Y Q (2013) A performance measure for Markov system with stochastic supply patterns and stochastic demand patterns, Reliability Engineering and System Safety 119: 294-299.

[27] Ackermann T, Andersson G, Söder L (2001) Distributed generation: a definition, Electric power systems research 57(3): 195-204.

[28] Blaabjerg F, Teodorescu R, Liserre M, Timbus A V (2006) Overview of control and grid synchronization for distributed power generation systems, IEEE Transactions on Industrial Electronics 53(5): 1398-1409.

[29] Hawkes A G, Jalali A, Colquhoun D (1990) The distributions of the apparent open times and shut times in a single channel record when brief events cannot be detected, Philosophical Transactions of the Royal Society of London - Series A 332(1098): 511-538. 